University of Nebraska - Lincoln

DigitalCommons@University of Nebraska - Lincoln

Ralph Skomski Publications

Research Papers in Physics and Astronomy

May 2002

\title{
Exchange-controlled magnetic anisotropy
}

Ralph Skomski

University of Nebraska-Lincoln, rskomski2@unl.edu

Follow this and additional works at: https://digitalcommons.unl.edu/physicsskomski

Part of the Physics Commons

Skomski, Ralph, "Exchange-controlled magnetic anisotropy" (2002). Ralph Skomski Publications. 16. https://digitalcommons.unl.edu/physicsskomski/16

This Article is brought to you for free and open access by the Research Papers in Physics and Astronomy at DigitalCommons@University of Nebraska - Lincoln. It has been accepted for inclusion in Ralph Skomski Publications by an authorized administrator of DigitalCommons@University of Nebraska - Lincoln. 


\title{
Exchange-controlled magnetic anisotropy
}

\author{
Ralph Skomski ${ }^{\text {a) }}$ \\ Department of Physics and Astronomy and Center for Materials Research and Analysis, \\ University of Nebraska, Lincoln, Nebraska 68588
}

The magnetocrystalline anisotropy of $5 f$ transition-metal atoms (actinides) is investigated. A simple model Hamiltonian reproduces the observed huge low-temperature anisotropy of cubic actinide compounds such as US and predicts the temperature dependence of the anisotropy. The dominance of the spin-orbit and crystal-field interactions means that the magnitude of the anisotropy is limited only by interatomic exchange. One consequence is that cubic and uniaxial $4 f$ magnets have similar magnitudes of the anisotropy and similar temperatures dependencies. (c) 2002 American Institute of Physics. [DOI: 10.1063/1.1451903]

\section{INTRODUCTION}

For many decades, advances in permanent magnetism have relied on the trend of realizing relativistic spin-orbit coupling in magnetic compounds and developing micromagnetically adequate morphologies. The result of that progress have been uniaxial rare-earth transition-metal magnets exhibiting impressive overall hard-magnetic properties. The first decades of the 20th century saw the development of uniaxial $3 d$-based magnets, such as $\mathrm{BaFe}_{12} \mathrm{O}_{19}$, where $K_{1}$ $=0.33 \mathrm{MJ} / \mathrm{m}^{3}$. The first 1:5 permanent magnets, developed in the late $1960 \mathrm{~s}$, were $\mathrm{YCo}_{5}$ magnets, ${ }^{1}$ whose anisotropy is due to the extraordinarily strong easy-axis contribution of the Co atoms in the 1:5 structure. ${ }^{2}$ Later, emphasis shifted toward $\mathrm{SmCo}_{5}$ and $\mathrm{Sm}_{2} \mathrm{Co}_{17}$ based sintered magnets, particularly for high-temperature applications, and to $\mathrm{Nd}_{2} \mathrm{Fe}_{14} \mathrm{~B} .{ }^{3}$ The success of the rare-earth transition-metal permanent magnets is closely linked to the anisotropy contribution of the rare-earth ions. ${ }^{2,4}$ This exploits the strong rare-earth spin-orbit coupling and leads to room-temperature anisotropies as high as $19 \mathrm{MJ} / \mathrm{m}^{3}$ for $\mathrm{SmCo}_{5}$. On the other hand, due to the small radius of the rare-earth $4 f$ shells, about $0.5 \AA$, the crystal-field interaction of the rare-earth $4 f$ electrons is quite small (about $0.01 \mathrm{eV}$ ). This prevents the rare-earth atoms from having even higher anisotropy.

The $5 f$ electrons of actinides, such as $\mathrm{U}$ and $\mathrm{Th}$, are metallic or - in compounds - at least close to being itinerant. Correspondingly, the crystal field acting on the $5 f$ electrons is much larger than $4 f$ crystal fields, and the strong spinorbit coupling is exploited quite efficiently. Indeed, some cubic actinide compounds, such as US, exhibit huge anisotropies on the order of $100-1000 \mathrm{MJ} / \mathrm{m}^{3}{ }^{5,6}$ However, the low Curie temperature of the materials prevents the exploitation of the high anisotropy at or above room temperature.

The present work investigates the magnetocrystalline anisotropy in the limit of strong spin-orbit coupling and strong crystal-field interaction, as realized in $5 f$ magnets. Particular emphasis is on the finite-temperature behavior of the anisotropy.

${ }^{\text {a)} E l e c t r o n i c ~ m a i l: ~ r s k o m s k i @ u n l s e r v e . u n l . e d u ~}$

\section{ORIGIN OF ANISOTROPY}

Magnetocrystalline anisotropy is the result of three fundamental interactions: spin-orbit coupling, crystal-field interaction, and interatomic exchange. Spin-orbit coupling is a higher-order relativistic correction to the Schrödinger equation. In the independent-electron approximation, it has the form $\mathcal{H}=\lambda \hat{\mathbf{l}} . \hat{\mathbf{s}}$, where $\hat{\mathbf{s}}$ is the spin, $\hat{\mathbf{l}}$ is the orbital momentum, and $\lambda$ is the one-electron spin-orbit coupling constant. For hydrogen-like atoms

$$
\lambda=\frac{m}{2} c^{2} \alpha^{4} \frac{Z^{4}}{n^{3} l(l+1 / 2)(l+1)},
$$

where $m$ is the electron mass, $c$ is the velocity of light, $\alpha$ $=1 / 137$ is Sommerfeld's fine-structure constant, $Z$ is the effective charge of the nucleus, and $n$ and $l$ are the principal and angular quantum numbers of the electron, respectively. Equation (1) shows that the spin-orbit coupling is particularly strong for the inner electrons in heavy elements, where the effective nuclear charge is large and the electrons move fast.

The spin-orbit coupling is isotropic with respect to a simultaneous rotation of $\hat{\mathbf{s}}$ and $\hat{\mathbf{l}}$, but the orbital motion of the electrons $\hat{\mathbf{l}}$ is affected by the anisotropic crystal field, for example by the $e_{g}-t_{2 g}$ splitting in cubic materials. ${ }^{7,8}$ From a basic point of view, there is no difference between the crystal-field splittings in insulators and metals. In insulators, the main contribution is from the electrostatic crystal field, ${ }^{9}$ whereas in metals the leading contribution is from linear combination of atomic orbitals (LCAO-type interatomic hybridization). ${ }^{10}$ Translated into band-structure theory, the electrostatic contribution corresponds to the on-site energy, whereas the hybridization contribution is due to interatomic hopping. ${ }^{11}$ The crystal-field interaction $\mathcal{H}_{\mathrm{CF}}$ determines the symmetry of the magnetocrystalline anisotropy and affects its strength.

Finally, the realization of magnetocrystalline anisotropy requires interatomic exchange. Without interatomic exchange $\langle\mathbf{s}\rangle=0$, and the electrons responsible for anisotropy do not couple to the spontaneous magnetization. This is particularly important at finite temperatures. 


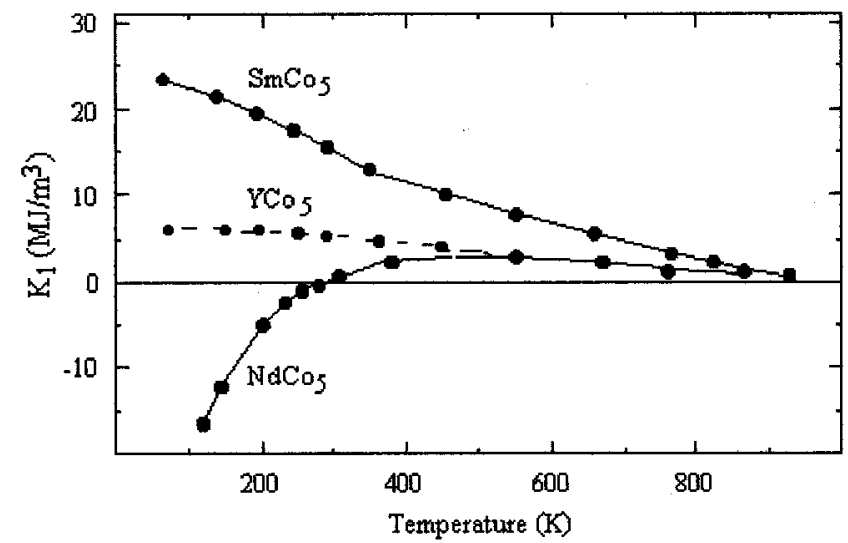

FIG. 1. Temperature dependence of the anisotropy of some rare-earth transition-metal intermetallics. Note that yttrium is a nonmagnetic' rare earth

\section{III. $3 d, 4 f$, AND $5 d$ MAGNETS}

In $3 d$-based magnets, such as $\mathrm{Fe}, \mathrm{YCo}_{5}$, and $\mathrm{BaFe}_{12} \mathrm{O}_{19}$, the anisotropy is limited by the quite weak $3 d$ spin-orbit coupling $\lambda \approx 0.05 \mathrm{eV}$, which competes against a strong crystal-field splitting ( $1 \mathrm{eV}$ or more). The analysis of the problem shows that the uniaxial anisotropy per atom as $\lambda^{2} / A$, where $A$ is the crystal-field splitting in insulators, or the bandwidth in metals. In cubic materials, the anisotropy is even lower, on the order of $\lambda^{4} / A^{3}$.

By contrast, in metallic and nonmetallic rare-earth magnets, the limiting parameter is the crystal-field interaction. Due to the rare-earths' large atomic weight, the spin-orbit coupling is sufficiently strong to create Hund's-rules atoms $(\lambda \approx 0.2 \mathrm{eV})$, but the small radius of the $4 f$ orbital leads to a very effective screening of the crystal field by conduction electrons $(0.02 \mathrm{eV}$ or less). Rare earths are therefore very ineffective in exploiting spin-orbit coupling. The exchange between the transition-metal sublattice and the rare-earth sublattice is moderately strong (about $0.01 \mathrm{eV}$ ). Figure 1 illustrates that this coupling is sufficient to create a large anisotropy at low temperatures, but above room temperature $K_{1}$ strongly decreases. The exchange between rare-earth atoms is even lower than the rare-earth transition-metal exchange and is usually neglected. In a sense, room temperature rare-earth anisotropy is created by the rare-earth sublattice but realized by the transition-metal atoms.

The anisotropy of $5 f$ magnets is determined by two factors. First, both the spin-orbit and the crystal-field interaction are very strong, on the order of magnitude of $1 \mathrm{eV}^{12}$ Second, the interatomic exchange tends to be very low, severely limiting the finite-temperature anisotropy. The relative strengths of the crystal-field and spin-orbit couplings may be estimated from the orbital moment, whose magnitude scales as $\lambda / A$. In uniaxial $3 d$ magnets, the orbital moment is on the order of $0.1 \mu_{B}$. In $5 f$ magnets, the orbital moment is comparable to the spin moment. For example, in US, a rocksalt ferromagnet with $T_{C}=177 \mathrm{~K}, \mu_{S}=3.5 \mu_{B}$, and $\mu_{L}=$ $-2 \mu_{B}$, so that $\mu \approx 1.5 \mu_{B}{ }^{6}$

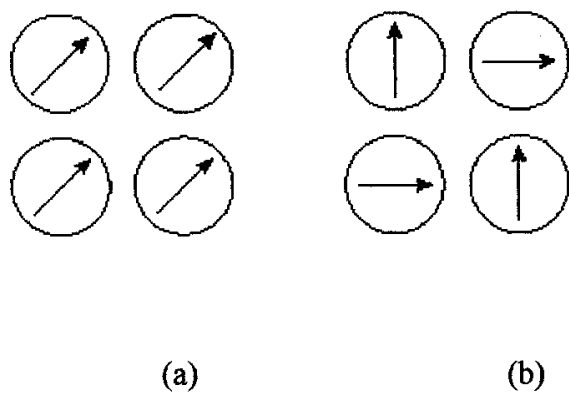

FIG. 2. Angular dependence of the anisotropy energy of $5 f$ magnets for cubic symmetry (solid line) and uniaxial symmetry (dashed line).

\section{MODEL AND CALCULATION}

To investigate the magnetocrystalline anisotropy contribution, we restrict ourselves to the ionic picture and start from the Hamiltonian

$$
\mathcal{H}=\mathcal{H}_{\mathrm{CF}}(\hat{\mathbf{L}})+\lambda \hat{\mathbf{S}} \cdot \hat{\mathbf{L}}+\mathbf{h}_{\mathrm{ex}} \cdot \hat{\mathbf{S}},
$$

where $\mathbf{h}_{\mathrm{ex}}$ is the interatomic exchange field. Since $\mathcal{H}_{\mathrm{CF}}$ and $\lambda$ dominate, the state of the ion is determined by the competition between crystal-field and spin-orbit interactions. Without spin-orbit coupling, the electrons adapt to the crystalline environment and tend to form standing waves with zero orbital moment (quenching), but the spin-orbit coupling introduces some circular current character. In any case, the outcome of the simultaneous diagonalization of $\mathcal{H}_{\mathrm{CF}}$ and $\lambda \hat{\mathbf{S}} . \dot{\mathbf{L}}$ is a set of well-defined quantum ground states $\left|\psi_{i}\right\rangle$. Here $i$ $=1 \ldots \mathrm{N}$ labels the quantization axes, which correspond to the $N$ crystallographically equivalent easy magnetization directions. For example, $N=2$ for uniaxial anisotropy and $N=6$ for cubic anisotropy with easy axes along the cube edges $\left(K_{1}>0\right)$. In lowest order perturbation theory, the anisotropy energy per atom is then given by

$$
E_{a}=\min _{j} \mathbf{h}_{\mathrm{ex}} \cdot\left\langle\psi_{i}|\hat{\mathbf{S}}| \psi_{i}\right\rangle,
$$

where $\mathbf{h}_{\text {ex }}$ points in the direction of the spontaneous magnetization.

To further evaluate Eq. (3), we have to specify the relative importance of the spin-orbit coupling. When $\lambda$ is small, the spin-orbit coupling acts a small perturbation to the crystal-field splitting (band structure). This limit is encountered in $3 d$ magnets, but also in $4 d$ and $5 d$ magnets such as $\mathrm{PdCo}$ and PtFe. In the present context, this case is not very interesting. The opposite limit, where the spin-orbit coupling dominates the crystal-field interaction, is also realized in $4 f$ intermetallics. However, in $4 f$ compounds the interatomic exchange is sufficiently strong to couple the rareearth atoms to the transition-metal sublattice, so that the anisotropy is essentially equal to the crystal-field interaction. $^{2-4}$ This mechanism breaks down when the crystal-field interaction exceeds the interatomic exchange. Figure 2 illustrates the difference by showing schematic spin structures for (a) rare-earth solids and (b) actinide solids.

In the limit of strong spin-orbit coupling, the minimization of Eq. (3) has a very simple meaning: spin and orbital moment are rigidly coupled and point in the easy direction 


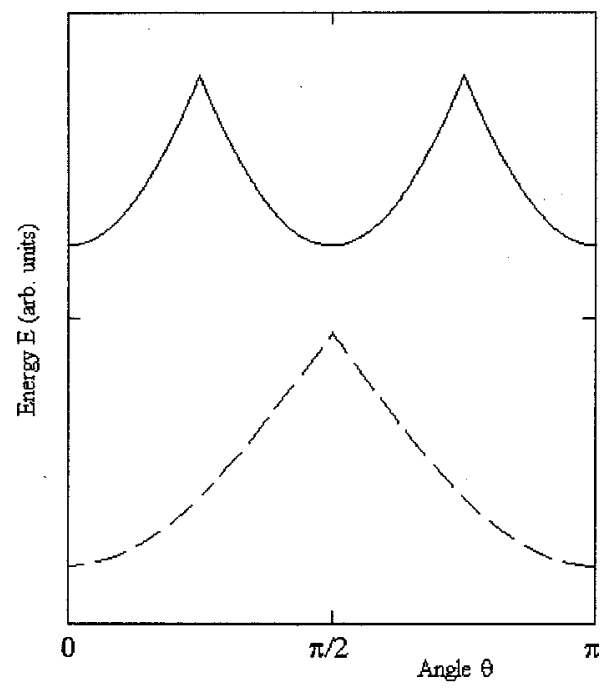

FIG. 3. Rotation of the magnetization of a sample: (a) strong interatomic exchange and (b) weak interatomic exchange. Simplifying somewhat, these two limits are realized in $3 d$ magnets and $5 f$ magnets, respectively.

whose projection onto $\mathbf{h}_{\mathrm{ex}}$ is largest. Figure 3 shows the exchange-controlled anisotropy energy $E_{a}(\theta)$ as a function of the magnetization angle $\theta$. Note that cubic and uniaxial anisotropies are very similar, except for a rather trivial geometrical factor.

\section{DISCUSSION AND CONCLUSIONS}

For small interatomic exchange, the leading contribution to the temperature dependence of the anisotropy is the reduction of $\mathbf{h}_{\mathrm{ex}}$. As a consequence, the temperature dependence of the anisotropy of actinides is magnetization-like, $K_{1}$ $\sim M$. By contrast, uniaxial and cubic $3 d$ magnets obey $M^{3}$ and $M^{10}$ laws, ${ }^{3,13}$ respectively, whereas the leading rare-earth anisotropy contribution in uniaxial $3 d-4 f$ intermetallics obeys a $1 / T^{2}$ behavior. ${ }^{14,15}$ Figure 4 compares these different temperature dependences.

Of course, the low Curie temperature of typical actinide magnets makes it difficult to realize this advantageous temperature dependence, but this does not necessarily exclude the possibility of room-temperature permanent magnets based on $5 f$ anisotropy. One may consider, for example, artificial structures where the actinide atoms have many transition-metal neighbors. In any case, the verification of nonanalytic energy landscapes such as those shown in Fig. 3 and their smoothing behavior in real magnets pose a challenge to experimental magnetism.

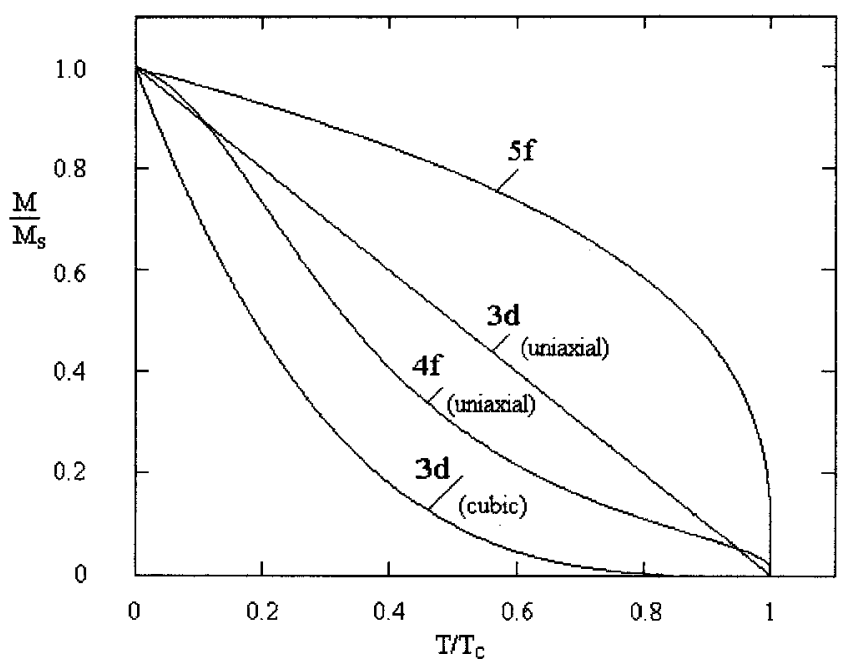

FIG. 4. Temperature dependence of the anisotropy (schematic).

In conclusion, we have shown that the large lowtemperatures anisotropies of $5 f$ magnets such as US are limited by interatomic exchange. Cubic and uniaxial exchangecontrolled anisotropies are of the same order of magnitude and exhibit similar temperature dependences. The anisotropies are characterized by a nonanalytic angular dependence and exhibit a quite weak temperature dependence below the Curie temperature.

\section{ACKNOWLEDGMENTS}

The author is grateful to Professor D. Leslie-Pelecky and Professor D. J. Sellmyer for stimulating discussions. This work has been supported by DOE, DARPA/ARO, and AFOSR.

${ }^{1}$ K. Strnat, Z. Kobalt 36, 119 (1967).

${ }^{2}$ J. P. Gavigan and D. Givord, J. Magn. Magn. Mater. 84, 288 (1990).

${ }^{3}$ R. Skomski and J. M. D. Coey, Permanent Magnetism (Institute of Physics, Bristol, 1999).

${ }^{4}$ J. M. Cadogan, J. P. Gavigan, D. Givord, and H. S. Li, J. Phys. F: Met. Phys. 18, 779 (1988).

${ }^{5}$ M. S. S. Brooks, B. Johansson, O. Eriksson, and H. L. Skriver, Physica B \& C 144, 1 (1986).

${ }^{6}$ M. S. S. Brooks and B. Johansson, Handbook of Magnetic Materials VII (Elsevier, Amsterdam, 1993).

${ }^{7}$ M. T. Hutchings, Solid State Phys. 16, 227 (1964).

${ }^{8}$ H. Bethe, Ann. Phys. (Leipzig) 3, 133 (1929).

${ }^{9}$ F. Bloch and G. Gentile, Z. Phys. 70, 395 (1931).

${ }^{10}$ C. J. Ballhausen, Ligand Field Theory (McGraw-Hill, New York, 1962).

${ }^{11}$ H. Brooks, Phys. Rev. 58, 909 (1940).

${ }^{12}$ P. Santini, R. Lémanski, and P. Erdös, Adv. Phys. 48, 537 (1999).

${ }^{13}$ H. R. Callen and E. Callen, J. Phys. Chem. Solids 27, 1271 (1966).

${ }^{14}$ M. D. Kuz'min, Phys. Rev. B 46, 8219 (1992).

${ }^{15}$ R. Skomski, J. Appl. Phys. 83, 6724 (1998). 